\title{
Trajectories of anxiety when children start school: the role of behavioural inhibition and attention bias to angry and happy faces
}

Article

Accepted Version

Dodd, H. F., Rayson, H., Ryan, Z., Bishop, C., Parsons, S. and Stuijfzand, B. (2020) Trajectories of anxiety when children start school: the role of behavioural inhibition and attention bias to angry and happy faces. Journal of Abnormal Psychology, 129 (7). pp. 701-712. ISSN 1939-1846 doi: https://doi.org/10.1037/abn0000623 Available at https://centaur.reading.ac.uk/92374/

It is advisable to refer to the publisher's version if you intend to cite from the work. See Guidance on citing.

To link to this article DOI: http://dx.doi.org/10.1037/abn0000623

Publisher: American Psychological Association

All outputs in CentAUR are protected by Intellectual Property Rights law, including copyright law. Copyright and IPR is retained by the creators or other copyright holders. Terms and conditions for use of this material are defined in the End User Agreement. 


\section{www.reading.ac.uk/centaur}

\section{CentAUR}

Central Archive at the University of Reading

Reading's research outputs online 
Author's own version of the following paper: Dodd, H.F., Rayson, H., Ryan, Z., Bishop, C., Parsons, S., \& Stuijfzand, B. (in press). Trajectories of anxiety when children start school: the role of Behavioural Inhibition and attention bias to angry and happy faces. Journal of Abnormal Psychology.

See: https://www.apa.org/pubs/journals/abn

Accepted for publication on $21 / 5 / 2020$

Author affiliations:

HD, ZR, CB: School of Psychology and Clinical Language Sciences, University of Reading, UK HR : Institut des Sciences Cognitives- Marc Jeannerod, CNRS / Université Claude Bernard Lyon, France

SP : Department of Experimental Psychology, University of Oxford, UK BS : Stuijfzand Data Consulting

Acknowledgements

Thank you to all of the Watch them Grow families for your time and support for our research. Thanks also to Hannah Stearne, Katy Whittle, Francesca Inzani, Charlotte LewisPryde, Charlie Murphy, Shellie May and Ola Lowicka for their help with the running of the project. The text message data collection was conducted by www.synapta.co.uk. The research was supported by an ESRC Future Leaders Fellowship awarded to HD (ES/L010119/1) and a Royal Society grant awarded to HD (RG2014R2). 


\begin{abstract}
Extensive research has examined attention bias to threat in the context of anxiety in adults, but little is understood about this association in young children and there is a dearth of longitudinal research examining whether attention bias to threat predicts anxiety over time in childhood. In the current study, a sample of 180 children participated in a longitudinal study, first as preschoolers and again as they transitioned to formal schooling. At baseline, children aged 3-4 years completed a free-viewing eyetracking task with angry-neutral and happy-neutral face pairs and an assessment of behavioural inhibition (BI). At follow-up, parents provided daily reports of their child's state anxiety over a 2-week period as their child started school and completed a measure of their child's anxiety symptoms. Results indicated that, on average, preschool-aged children exhibit a bias for emotional faces that is stronger for angry than happy faces. There was little evidence that this bias was associated with anxiety symptoms. However, BI interacted with dwell bias for angry faces to predict trajectories of anxiety over the transition to school. An unexpected interaction between $\mathrm{BI}$ and dwell bias for happy faces was also found, with dwell for happy faces associated with lower anxiety for children higher in BI. The findings are consistent with recent developmental models of the $\mathrm{BI}$-anxiety relationship and indicate that attention bias modification may not be suitable for young children, for whom attention bias to threat may be normative.
\end{abstract}

Keywords: Anxiety, children, attention-bias, emotional faces, transition to school.

\title{
General Scientific Summary
}

Pre-school predictors of children's anxiety symptoms and state anxiety (reported daily) over the transition to school at age 4 were examined. Specifically, attention biases to angry and happy faces, relative to neutral, and an avoidant temperament style known as behavioural 
inhibition (BI) were examined as predictors. $\mathrm{BI}$ predicted anxiety symptoms and state anxiety. There was no evidence that attention biases directly predicted anxiety, although biases interacted with temperament to predict trajectories of state anxiety over the transition. 
Early cognitive models defined biased attention as a central mechanism underpinning anxiety (Beck \& Clark, 1997; Mathews \& Mackintosh, 1998; Williams, Watts, MacLeod, \& Mathews, 1988) and extensive research has evaluated this hypothesis. There is some evidence for an anxiety-linked attention bias for threat (Bar-Haim et al., 2007) although this is not consistently found (Kruijt, Parsons, \& Fox, 2018) and there are concerns about the reliability of attention bias tasks (Rodebaugh et al., 2016). In attention bias theory and research, anxiety is typically defined broadly to include clinical and non-clinical samples as well as state and trait anxiety. This broad definition of anxiety also aligns with The National Institute of Mental Health Research Domian Criteria (RDoC; National Insititute of Mental Health, 2011) and theoretical ideas about the relationship between anxiety disorders, trait and state anxiety (e.g. Raymond, Steele \& Series, 2017).

Over the past two decades there has been growing interest in anxiety-linked attention bias in children. Using reaction-time tasks analogous to those used with adults, some developmental studies support an anxiety-linked bias (e.g. Abend et al., 2018), but there are inconsistencies. For instance, biases away from threat have been found in certain anxiety disorders (Salum et al., 2013; Waters, Bradley, \& Mogg, 2014) and some studies find no association between anxiety and attention bias in childhood (e.g. Britton et al., 2013). A recent meta-analysis including studies of clinically diagnosed and community samples found that anxiety is associated with an attention bias for threat in children, but this effect is smaller than that found in a meta-analysis of the adult literature (Bar-Haim et al., 2007) and is weaker in younger children (Dudeney et al., 2015).

Despite this increased interest, there remains a dearth of research focused on anxiety-linked attention bias in young children; the lowest average age of participants in studies included in the Dudeney et al (2015) meta-analysis was 9 years. Assessing bias in 
young children is challenging due to a reliance on reaction-time tasks. Such tasks are problematic because motor responses are distal from attentional processing, and young children have slower and more variable motor functioning (Price et al., 2015; Van Damme \& Crombez, 2009). Indeed, Brown et al. (2014) showed that reaction-time based measures of attentional bias have poor reliability in children. In contrast, eyetracking can have better accuracy and reliability (Price et al., 2015; Waechter, Nelson, Wright, Hyatt, \& Oakman, 2014), although this is not consistently found and can vary depending on the metric extracted from the gaze data (Skinner et al., 2018). Eyetracking also has the advantage that it can be used in free-viewing paradigms that do not require complex instructions.

Eyetracking has successfully been employed to investigate patterns of anxiety-linked attention bias in children. Some of this research suggests that anxious youth exhibit an orientation bias for angry versus neutral faces (Shechner et al., 2013), but other studies have reported more nuanced results (Schmidtendorf, Wiedau, Asbrand, Tuschen-Caffier, \& Heinrichs, 2018) or effects that vary by age (Gamble \& Rapee, 2009). A recent meta-analysis concluded that eyetracking studies with children and adolescents do not support an anxietylinked vigilance bias for threat (Lisk, Vaswani, Linetzky, Bar-Haim, \& Lau, 2019). In the only study to have examined anxiety-linked biases specifically in young children, both anxious and non-anxious 3-4 year olds exhibited orientation and dwell biases for angry over neutral faces, with no between group differences (Dodd et al., 2015).

Various theories have been proposed regarding the role of attention bias in anxiety (see Mogg \& Bradley, 2016 for a review). Across these theories two differing perspectives emerge (Mogg \& Bradley, 2018). Some theorists argue that attention bias is relatively stable and plays a causal role in anxiety (MacLeod \& Clarke, 2015), acting as a vulnerability factor that leads to elevated anxiety when a stressful event occurs (Mathews \& MacLeod, 2002). 
Others argue that attention bias may not play a causal role in anxiety, instead acting as a maintenance factor (Mogg \& Bradley, 1998).

Two main lines of adult research have investigated whether attention bias plays a causal role in anxiety: attention bias modification ( $A B M)$ studies and longitudinal studies (see Van Bockstaele et al., 2014 for a review). In support of the causal hypothesis, several studies suggest that ABM can decrease anxiety in adults (Bar-Haim, 2010) and youth (Pettit et al., 2019). However, notable inconsistencies across studies exist and the debate about whether ABM can successfully decrease anxiety symptoms continues (Cristea, Kok, \& Cuijpers, 2015; Grafton et al., 2017; Mogg, Waters, \& Bradley, 2017). Longitudinal research with adults also provides support for the causal hypothesis. For example, MacLeod and Hagan (1992) found that attention to threat-related words predicted emotional distress in women following a medical diagnosis. Similar findings have been reported in other adult populations, such as in the study by Van Den Hout and colleagues (1995), in which attentional bias was a significant predictor of reactions to life stress in healthy participants, and was a better predictor than trait anxiety. A crucial feature of these longitudinal studies is that they examine state responses to a stressor. This is relevant to anxiety disorders because it is theorised, and supported by the evidence extant, that anxiety disorders involve a primary malfunction in the brain's ability to regulate anxious states (Raymond et al., 2017). Therefore, studying state anxiety in response to a stressor is relevant for understanding risk for anxiety disorders.

Longitudinal research examining whether attention bias predicts anxiety in children is rare and, to our knowledge, has not examined response to a stressor. In developmental research, attention bias has typically been examined alongside the temperament style Behavioural Inhibition (BI). Children high in $\mathrm{Bl}$ are characterised by withdrawal and wariness 
in unfamiliar, novel situations (Kagan, Reznick, Clarke, Snidman, \& Garcia-coll, 1984).

Extensive evidence suggests that $\mathrm{Bl}$ is a predictor of subsequent social reticence and anxiety (Chronis-Tuscano et al., 2009; Claus \& Blackford, 2012; Hudson, Murayama, Meteyard, Morris, \& Dodd, 2018). However, not all children who are high in BI go on to experience difficulties with anxiety (Fox, Henderson, Rubin, Calkins, \& Schmidt, 2001) and various moderators of the BI-anxiety relationship have been proposed (Degnan \& Fox, 2007) including individual differences in cognitive processing biases, such as attentional bias (Liu \& Pérez-Edgar, 2019).

Emerging research provides fairly consistent evidence that child temperament and attention bias interact to affect anxiety and social withdrawal (Cole, Zapp, Fettig, \& PérezEdgar, 2016; Morales, Pérez-Edgar, \& Buss, 2015). Perez-Edgar et al. (2010) found that BI in early childhood predicted adolescent social withdrawal only when adolescents also had an attention bias to threat. These findings were subsequently supported in younger children, with $\mathrm{BI}$ in early life predicting social withdrawal at age 5 only in those with concurrent attention bias (Perez-Edgar et al., 2011). Two follow-up assessments of this same sample revealed similar patterns for trait anxiety at ages 7 (White et al., 2017) and 10 (Nozadi et al., 2016). Such findings indicate that attention bias may be a core mechanism sustaining $\mathrm{BI}$ over development, resulting in elevated risk for social reticence and anxiety (Henderson, Pine, \& Fox, 2015). To our knowledge, the studies conducted by White et al. (2017) and Nozadi et al. (2016) are the only longitudinal studies that have evaluated whether attention bias predicts anxiety over time in children, and importantly, neither considered children's state anxiety in response to a stressor. Furthermore, these studies relied on reaction-time based measures of attention bias. 
The present research has three aims. First, to evaluate whether attention bias to threat and BI predict children's concurrent anxiety symptoms; second, to evaluate whether attention bias to threat and BI predict children's anxiety symptoms prospectively; and third, to examine whether attention bias to threat and BI predict children's state anxiety in response to a stressor. We make use of starting school as a naturalistic stressor, hypothesising that: anxiety trajectories over the transition to school, as well as anxiety symptoms at baseline and follow-up, will be predicted by BI and by an attention bias for angry faces, and that attention bias for angry faces will interact with $\mathrm{BI}$ to predict anxiety.

\section{Method}

\section{Participants}

A sample of 180 typically developing children aged $3.42-4.83$ years $(M=3.97, S D=$ 0.25; 90 female) were recruited via preschools, public advertising, social media and word of mouth to take part in a project about children's emotions when they start school. Most children were described by their parent as being White British (83.3\%). See S1 for power analysis details and further sample information.

Follow-up took place as children started school. The average time between baseline and starting school was 6.19 months ( $S D=2.25$ months; range $3-11$ months).

\section{Apparatus and Materials}

Parent report of BI. The Behavioural Inhibition Questionnaire (BIQ; Bishop, Spence, \& McDonald, 2003) is a parent-report measure with 30 items. Parents are asked to indicate how often each behavior occurs for their child. The measure has good psychometric properties, adequate internal consistency, moderate stability of time and strong construct validity (Bishop et al., 2003; Kim et al., 2011). In this sample, Cronbach's alpha = .96. 
Observation of BI. The two fear episodes most relevant to BI (Risk Room and Stranger Approach) from the Laboratory Temperament Assessment Battery (Lab-TAB) were used. The Lab-TAB is a standardised observational measurement of temperament designed for use with 3-5 year old children (Gagne, Van Hulle, Aksan, Essex, \& Goldsmith, 2011). Further details of the procedure for these episodes can be found in the supplementary material and Lab-TAB manual (Goldsmith, Reilly, Lemery, Longley, \& Prescott, 1999).

Episodes were videotaped and coded according to the Lab-TAB manual. Scores for each coding criteria were reverse coded as necessary so that higher scores indicated more inhibited responses. These scores were then converted into z-scores individually and then averaged into a single $\mathrm{BI}$ score $(\mathrm{M}=-0.04, \mathrm{SD}=0.44$, range $=-0.74-1.53)$ in line with previous research (Gagne et al., 2011; Goldsmith et al., 1999). To check reliability a secondary coder coded $24 \%$ of the Lab-TAB assessments. Interrater reliability was good to excellent $\operatorname{ICC}(2,1)$ $=.95 ; 95 \% \mathrm{Cl}=.91$ to .98$)$. Both coders were blind to the child's anxiety scores and bias scores.

Overall BI score. Children's scores on the BIQ and their observed BI scores were correlated $(r=.32, p<.001)$ and were combined by converting both measures into $z$-scores and averaging. For 17 participants this combined score was missing due to technical problems whilst completing the Lab-TAB.

Parent report of anxiety symptoms. The Preschool Anxiety Scale (PAS; Spence, Rapee, McDonald, \& Ingram, 2001) is designed for parents of children aged 2.5-6.5 years and the total score provides an overall measure of child anxiety symptoms (or trait anxiety). The measure has good construct validity, satisfactory internal consistency and good crossinformant and test-retest reliability (Spence et al., 2001). Cronbach's alpha at both baseline 
and follow-up $=.91$. There was no missing data at baseline but 16 participants were missing follow-up PAS scores. These children did not differ from those with PAS scores on anxiety, age, gender, ethnicity, parent marital status or parent employment status.

Parent report of daily anxiety during transition to school. State anxiety ratings were obtained via text messages sent daily to parents over the transition to school period. The text message read: Please reply with your rating for [Child's name]'s anxiety today. Using a scale from 0 to 10, with 0 indicating no anxiety or worries at all and 10 indicating extremely anxious/worried. Parents were also asked to reply indicating whether their child had attended school that day and how much of a problem their child's anxiety had been. For the latter, the responses were very highly correlated with the level of anxiety $(r=.83)$ so the focus here is on anxiety ratings only. Construct validity of this measure is provided by correlations between average anxiety score reported via text and PAS total score at both baseline and follow-up (see Table 1). At least 7 days of ratings were provided for 179 of the 180 participants, 150 responded on all 14 days. Overall, only $2.5 \%$ of anxiety ratings were missing. The mean number of days that ratings were given was $13.56(S D=1.19 ;$ Minimum $=$ 7 days).

\section{Attention Bias Task}

Eyetracking apparatus. Eye movements were measured using a remote deskmounted Tobii T300 eyetracker. The task was programmed in e-prime version 2.0.10.356 (Psychology Software Tools, Pittsburgh, PA) and presented on a Viewsonic VA2413wm 24" monitor with $1920 \times 1080$ resolution. The e-prime script is available from the corresponding author. 
Face stimuli. Colour photographs of 16 Caucasian child actors ( 8 male and 8 female), each displaying neutral, angry and happy facial affect, were sourced from the Child Affective Facial Expression set (CAFE; LoBue \& Thrasher, 2015). Happy faces were included as well as angry faces to ensure any observed effects could be attributed to the threatening, angry faces specifically as opposed to emotional faces in general. The faces chosen had the highest available accuracy ratings. The CAFE images are aligned based on each actor's individual eye alignment. The photographs were manipulated so that all images from the same actor were matched for RGB values as well as mean and standard deviation luminance. Photographs were resized to $178 \times 187$ at $60 \mathrm{ppi}$. The face stimuli were positioned to centre at $761 \times 540$ and 1159x540. The viewing distance was approximately $65 \mathrm{~cm}$, giving a viewing angle of 4.4 degrees to the centre of the screen, and 9.4 degrees between the centre points of the face stimuli.

Design. The task began with a 9-point eyetracker calibration. Each trial began with a fixation screen for 500 ms showing a central cross surrounded by a 2 " square purple outline to highlight the fixation cross. Gaze contingency was used such that the experimental screen began only after the participant had fixated within the square for a minimum of $100 \mathrm{~ms}$. The fixation screen was then replaced by the experimental screen for $1500 \mathrm{~ms}$. The experimental screen consisted of an emotion (happy/angry) and neutral face displayed side by side of the same child (positions counterbalanced). There were 64 trials (32 happy-neutral; 32 angryneutral).

To make the task engaging for children, on 15 additional trials a cartoon monkey was presented centrally. Fifteen images were used, each showing the monkey in a different position (hanging from a branch, sitting arms crossed etc.). The ordering of experimental 
and monkey screens was randomized. Monkey screens remained until the experimenter pressed a resume button.

Eyetracking data. Eye movement data was continuously recorded during stimulus presentation $(1500 \mathrm{~ms})$ at a sampling rate of $300 \mathrm{~Hz}$. This means that the position of the left and right eye was recorded every $3.33 \mathrm{~ms}$. Each of these samples was then processed in relation to three Areas of Interest (AOIs) defined based on the location of the two face images and the square surrounding the fixation cross.

The raw eyetracking data were processed in R version 3.4.2 using EyetrackingR (Dink \& Ferguson, 2018). Four participants did not start the task due to problems calibrating and three were excluded because they did not complete the task. Fixation plots were visually inspected and five additional participants were excluded given evidence of calibration drift. Data was therefore available for $168 / 180$ participants (93\%). Individual trials were removed if there was greater than $40 \%$ trackloss for that trial. On average, the included sample had 59.3 valid trials and $<8 \%$ trackloss. Excluded participants did not differ significantly from included participants on age, sex, ethnicity, BI score, baseline PAS score, follow-up PAS score or average anxiety rating (all $p^{\prime} s>.1$ ). The following bias scores were calculated for use in the analyses:

1. Orientation bias. Orientation bias for angry faces was calculated as the percentage of angry-neutral trials where participants looked to a face, in which gaze was recorded within the angry face $\mathrm{AOI}$ first. The equivalent was also calculated for happy faces. The split half estimate of reliability was calculated using splithalf in R (Parsons, 2019; Parsons, Kruitj, \& Fox, 2019) and was $r_{s b}=-.18$, $95 \% \mathrm{Cl}[-.34-.01]$ for bias to angry faces and $r_{\mathrm{sb}}=-.33,95 \% \mathrm{Cl}[-.46--.17]$ for bias 
to happy faces. Given these poor reliability estimates, no analyses are conducted using orientation bias.

2. Dwell bias. The position of participant gaze was sampled and recorded every $3.33 \mathrm{~ms}$, or at $300 \mathrm{~Hz}$. To calculate dwell bias for angry faces, the number of samples recorded in the angry face $\mathrm{AOI}$ and neutral face $\mathrm{AOI}$ during angry-neutral trials were calculated as a proportion of the total number of on screen samples recorded during each trial, and the resulting proportion of looking to the neutral face was subtracted from proportion of looking to the angry face. The equivalent was calculated for happy dwell bias using happy-neutral trials. The split half estimate of reliability was calculated using splithalf in R (Parsons, 2019; Parsons, Kruitj, \& Fox, 2019) and was $r_{\mathrm{sb}}=.63,95 \% \mathrm{Cl}[.53-.71]$ for bias to angry faces and $r_{\mathrm{sb}}=.39,95 \% \mathrm{Cl}[.23-.53]$ for bias to happy faces.

\section{Procedure Baseline}

Procedures were approved by the University of Reading Research Ethics committee (UREC 16/56). Informed consent and assent were obtained from parents and participants after being provided with information about the project. Families were invited to a lab session during which they completed the tasks reported here, as well as other tasks forming part of a larger longitudinal study. The Lab-TAB was completed prior to the eyetracking task. For the eyetracking task, participants were told that they were going to play a game using a computer that knew where they were looking. They were given a sheet displaying the 15 cartoon monkeys and were told they needed to look out for each one on the screen. Participants were told that lots of faces would also appear on the screen. One of the researchers sat with the participant throughout the task to encourage engagement and 
provide support with the monkey checklist. At the end of the lab session participants were thanked for their participation and given a small gift; parents were given $£ 35$.

\section{Procedure follow-up}

Parents consented to take part in the follow-up stage of the research during the baseline session. The follow-up stage included 14 days of daily ratings of anxiety obtained via text message and online questionnaires. Text messages were sent every evening at 7:30pm beginning two days before the child's first day at school. Reminder texts were sent the following morning if no response was received. Parents were emailed approximately one week after their child started school asking them to complete a series of questionnaires online. A reminder email was sent two weeks later, and the online questionnaire was closed approximately 6 weeks after the children's first day at school.

\section{Missing data}

Full details regarding missing data are shown in the supplementary materials. Missing data was handled using mice (van Buuren \& Groothuis-Oudshoorn, 2011) meaning that all participants and available data were included in analyses. In total, only $6 \%$ of all data was missing at baseline. At follow-up, $9 \%$ of PAS anxiety scores were missing and $2.5 \%$ of text message daily ratings were missing. In terms of participant numbers, 152 had complete data at baseline. Of these, 137 had PAS follow-up data (76\% of the sample) and all had $>7$ days of state anxiety ratings.

\section{Data analysis plan}

Analyses were conducted in R version 3.5.3 using the tidyverse suite (Wickham, 2017). 
No potential confounds were identified. Linear regression was used to examine whether $\mathrm{BI}$ and attention bias predict PAS anxiety scores at baseline, addressing aim 1, and at follow-up, addressing aim 2. Growth Curve Analysis (GCA) was then used to examine whether $\mathrm{BI}$ and attention bias predict trajectories of anxiety over the transition to school, addressing aim 3. All variables were centered prior to analysis.

\section{Assumption checks}

For all variables, distributions were evaluated and checks for univariate and multivariate outliers were conducted. Two issues were found. First, the anxiety rating data was negatively skewed; $44 \%$ of the 2520 data points were zeros meaning that nonlinear transformations did not lead to a distribution that was approximately normal. Consequently, for the GCAs involving this variable, Robust Maximum Likelihood Estimators were used. One participant was an outlier on the anxiety ratings measure but their inclusion did not affect the pattern of results so they are included.

\section{Results}

The dataset and analysis code are available to download, along with other measures collected as part of the wider study, at: http://reshare.ukdataservice.ac.uk/853813/. Given the poor reliability of the orientation bias variables, no analyses are conducted using these variables. The results focus only on dwell bias.

Table 1 shows the descriptive statistics for each of the variables and the correlations between variables. Anxiety symptoms at baseline and follow-up were aligned with PAS norms for 3 and 4 year olds (norms as follows: $M=22.86, S D=15.57 ; M=18.81, S D=13.90$ respectively) indicating that anxiety levels of the sample were representative of the general population. Anxiety symptoms were highly correlated and both were correlated with the 
average daily anxiety rating. BI was associated with anxiety at baseline and follow-up as well as average daily anxiety rating. There was little evidence for a direct association between attention bias and anxiety. Table 1 shows that dwell bias for happy faces and for angry faces were significantly correlated. Given this, we will examine whether findings are consistent with both dwell bias variables in the same model after evaluating the effects for happy and angry faces separately.

\section{Characterisation of attention bias}

One sample $t$-tests using complete data indicated that the sample as a whole exhibited a significant dwell bias to both angry, $\mathrm{t}(167)=23.37, p<.001,95 \% \mathrm{Cl}=0.13-0.15$, $d=1.80$, and happy, $\mathrm{t}(167)=5.94, p<.001,95 \% \mathrm{Cl}=0.019-0.04, d=0.45$, faces. Bias for angry was significantly stronger than the bias for happy, $\mathrm{t}(331.71)=5.03, p<.001$.

\section{Predicting anxiety symptoms at baseline}

Linear models were conducted with 20 imputed datasets. Anxiety symptoms at baseline was the outcome variable, and attention bias and BI, together with their interaction, were predictor variables. Two models were conducted for angry and happy, dwell bias (Table 2). Across all models BI was a significant predictor $(p<.001)$. There were no significant main effects of the attention bias variables. The interaction between dwell bias for happy faces and $\mathrm{BI}$ approached significance $(p=.06)$. When dwell bias for angry faces and dwell bias for happy faces were included in the same regression model (Table 2) there were no significant main effects or interactions that included the attention bias variables, although the BI by dwell bias for angry faces approached significance $(p=.06)$.

\section{Predicting anxiety symptoms at follow-up}


Equivalent linear models were conducted with anxiety symptoms at follow-up as the outcome variable (Table 3). The pattern of results was identical to baseline. Across all models BI was a significant predictor $(p<.001)$. There were no significant main effects of the attention bias variables and no significant interactions between $\mathrm{BI}$ and attention bias, although the interaction between dwell bias for happy faces and $\mathrm{BI}$ was significant $(p=.04)$. A small negative association between dwell bias for happy faces and anxiety was apparent but only for children higher in BI. When dwell bias for angry faces and dwell bias for happy faces were included in the same regression model (Table 3) there were no significant main effects or interactions that included the attention bias variables $(p>.08)$.

\section{Predicting anxiety over the transition to school}

We examined whether $\mathrm{BI}$ and dwell bias to angry and happy faces were predictors of anxiety trajectories over the transition to school period using GCA with the Ime4 (Bates, Mächler, Bolker, \& Walker, 2014) and ImerTest (Kuznetsova, Brockhoff, \& Christensen, 2017) packages. $R^{2}$ estimates were calculated using the multilevelR2 function in the mitml package in R (Grund, Robitzsch, \& Luedtke, 2019). Data from 20 imputed datasets were pooled to give the results reported. The model selection process is outlined in the supplementary material. The final models and results are shown in Tables 4, 5 and 6. Across models there was consistently a significant main effect of $\mathrm{BI}$, as expected; children higher in $\mathrm{BI}$ had greater anxiety scores over the transition to school. There was also a significant linear effect of time in all models; over time children's anxiety decreased.

Angry faces. For dwell bias for angry faces (Table 4) no main effect was found but there was a significant three-way interaction between dwell bias for angry faces, BI and quadratic time $(p=.005)$. This interaction is shown in Figure 1 . The figure suggests that attention bias affected the pattern of anxiety over time for the High BI children, with High $\mathrm{BI}$ 
children who also had an attention bias to threat having the highest levels of anticipatory anxiety during the two days before starting school, which declined quite rapidly once they started school. In contrast, High BI children with a relatively low attention bias to threat showed a slight increase in anxiety as they started school, which began to decline after the first few days at school. For Low BI children, those who also had a relatively low attention bias to threat showed higher levels of anxiety overall and particularly in advance of starting school, compared to those who had a higher attention bias to angry faces.

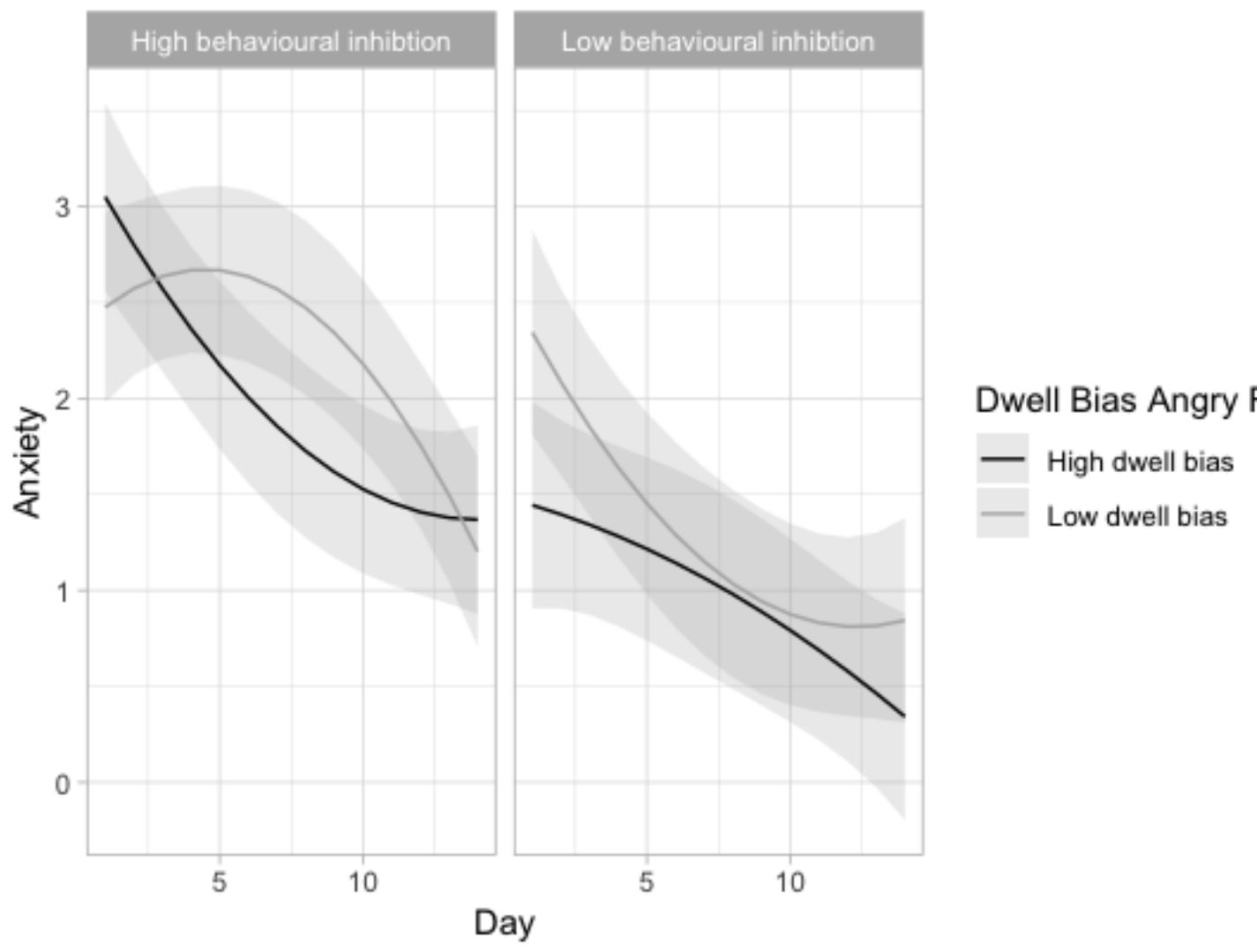

Figure 1: Interaction between dwell bias to angry faces, behavioural inhibition and quadratic time. Lines indicate high and low orientation bias and the two plots indicate high and low $\mathrm{Bl}$; both operationalized as one SD above and below the respective mean. Shading shows $95 \%$ confidence intervals. 
Happy faces. For dwell bias to happy faces (Table 5) there was a significant main effect of $\mathrm{BI}(p<.001)$ and a significant main effect of linear time $(p<.001)$. There was no significant main effect of dwell bias to happy faces, but there was a significant interaction between $\mathrm{Bl}$, dwell bias to happy faces and linear time $(p=.001)$. This interaction is shown in Figure 2, which indicates that High BI children who had a lower dwell bias to happy faces had the highest levels of anxiety in anticipation of starting school, but then showed a steeper decline relative to other groups.

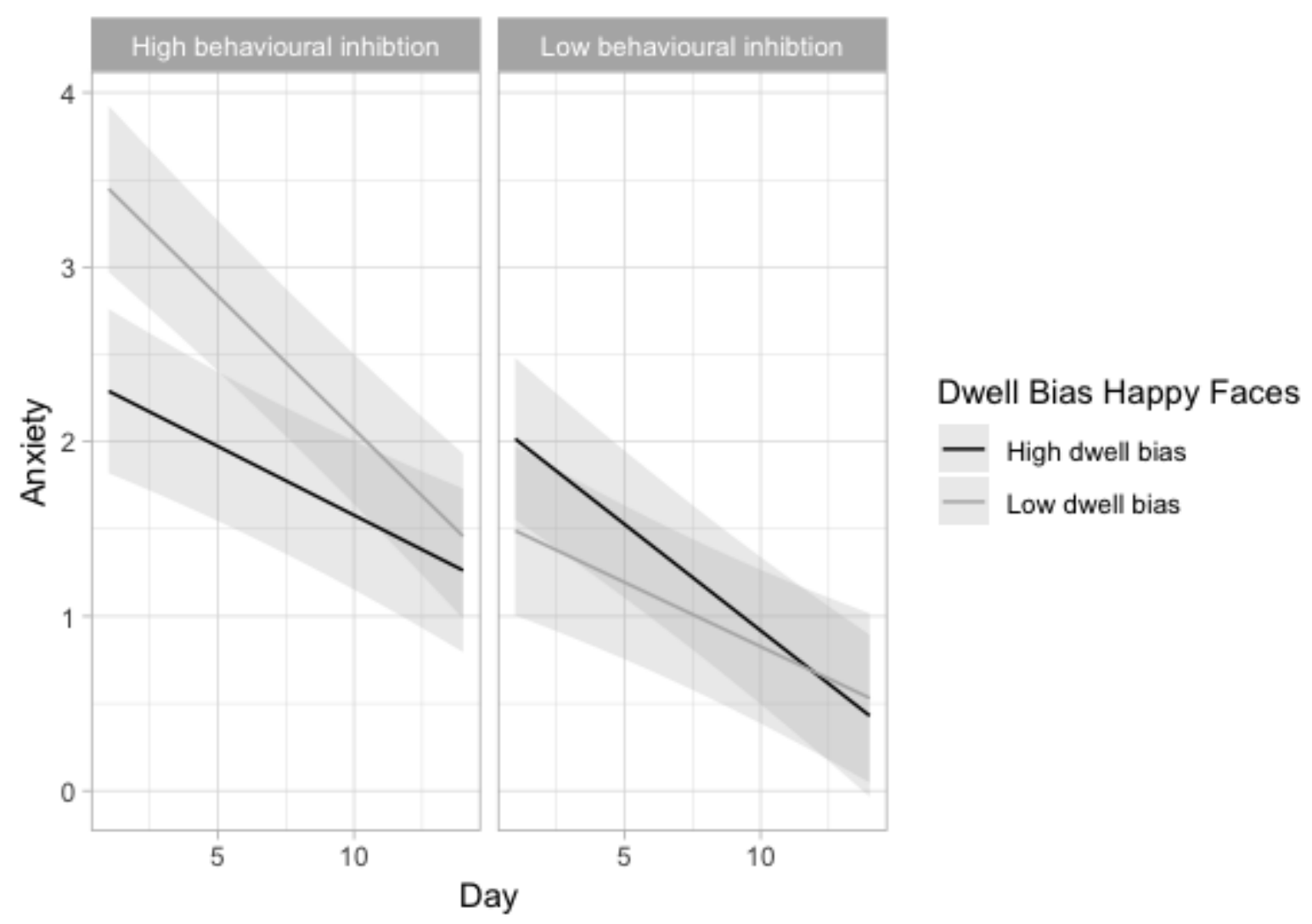

Figure 2: Interaction between dwell bias to happy faces, behavioural inhibition and linear time. Lines indicate high and low dwell bias and the two plots indicate high and low BI; both operationalized as one SD above and below the respective mean. Shading shows $95 \%$ confidence intervals. 
Happy and angry faces. When dwell bias to angry faces and dwell bias to happy faces were included in the same model (Table 6), the interactions observed in the separate models remained significant but no other main effects or interactions that included the attention bias variables were found.

\section{Discussion}

We evaluated whether, in young children, attention bias for threat is associated with trait anxiety and state anxiety in response to a stressor. In line with previous developmental work and theoretical models, attention bias was examined together with BI. Given poor split-half reliability estimates for the orientation bias variables, the results are based on dwell bias only. As expected, BI was a robust, significant predictor of anxiety symptoms at baseline and follow-up, as well as state anxiety over the transition to school. Contrary to our hypotheses, there was little evidence for a direct association between attention bias to angry or happy faces and anxiety, and no evidence for an interaction between $\mathrm{BI}$ and attention bias to threat in predicting anxiety symptoms at baseline or follow-up. Nevertheless, the hypothesis that $\mathrm{BI}$ and attention bias to threat would interact to predict trajectories of state anxiety over the transition to school was supported. We also found an unexpected interaction between dwell bias to happy faces and BI in predicting state anxiety.

On average, children exhibited a bias for both angry and happy faces. This bias for angry faces fits with a large body of developmental research demonstrating that an attention bias to threat is normative and present from infancy (e.g. LoBue, 2009; LoBue \& DeLoache, 2010). The bias for happy faces was subtle but is in keeping with recent evidence that attention is focused on happy faces (Bucher \& Voss, 2019). Bias for angry faces and bias for happy faces were positively correlated, which suggests that at least some children may exhibit an underlying bias for emotional faces in general. Given this, we evaluated whether 
the results remained the same when happy and angry bias were included in the same model. For the state anxiety analyses the results were identical, which allows us to confidently interpret these findings as being specific to each emotion.

Overall there was little evidence that 3-4 year old children have an anxiety-related attention bias, which is consistent with previous eyetracking research with young children (Dodd et al., 2015) and the conclusion of a recent meta-analysis of eyetracking studies in children and adolescents (Lisk et al., 2019). A bias to threat early in life without clear evidence of an anxiety-linked bias is consistent with what Field and Lester (2010) term the moderation model, where attention bias to threat is normative in young children and the association between bias and anxiety emerges with development. This of course assumes that an anxiety-linked bias is reliably present in older children and adults. Whilst metaanalyses of attention bias studies have supported this link with anxiety (e.g. Bar-Haim et al., 2007; Dudeney et al., 2015), a recent meta-analysis of data that are arguably less likely to be affected by publication bias, suggests that anxiety is not characterized by attention bias towards threat (Kruijt et al., 2018). Our results are also consistent with this conclusion.

State anxiety in response to a stressor is considered a vulnerability marker for subsequent trait anxiety and anxiety disorders (Raymond et al., 2017) and is therefore relevant for identifying potential risk factors for anxiety disorders. Our results showed that $\mathrm{BI}$ interacted with attention bias to threat to predict anxiety trajectory across the transition to school. For children with high BI, having a high dwell bias to angry faces predicted high anticipatory anxiety followed by a steep decline over time. A similar pattern was seen for children with low BI, albeit at a lower overall level of anxiety, but in those who had a low dwell bias to angry faces. In comparison, children with high BI with a low dwell bias showed a slight increase in anxiety over the first days at school, followed by a shallower decline. A BI 
by threat bias interaction is in keeping with previous work (e.g. Perez-Edgar et al., 2011; White et al., 2017), but our findings differ because attention bias was not a clear predictor of anxiety level. Instead, attention bias affected anxiety trajectory over time differently depending on level of BI.

Notably, for children who were relatively low in $\mathrm{BI}$, having an attention bias for angry faces predicted less anxiety over the transition to school. We are reluctant to overinterpret this unpredicted finding, but this may indicate that attention bias to threat is adaptive for children who are generally disinhibited and take more risks. Alternatively, a combination of a threat-related attention bias and uninhibited behavior could mean that these children notice potential threats and, by approaching them with curiosity, quickly learn whether they are associated with negative outcomes or not.

We also found an unexpected interaction between happy dwell bias and BI predicting anxiety trajectory over the transition to school; dwell bias to happy faces was associated with lower levels of anxiety in children who were high in BI. The interaction remained significant after controlling for the effects of dwell bias to angry faces, which suggests that maintained attention to positive social stimuli might serve as a protective factor against state anxiety for children high in BI. This finding must be interpreted cautiously given it was not hypothesized and the split-half reliability for the happy dwell bias score was relatively low. The result is however consistent with a reanalysis by Shechner et al. (2012) using data from Perez-Edgar et al. (2011), which showed that children who were high in $\mathrm{BI}$ and had a bias to happy faces had low anxiety relative to children who were high in $\mathrm{BI}$ without a bias to happy faces. Furthermore, work with children raised in institutions has shown that an attention bias for positive stimuli is associated with stable foster care placement, fewer internalizing symptoms and better coping mechanisms (Troller-Renfree et al., 2017). ABM 
work has also shown that training a happy face bias significantly reduces anxiety severity in clinically anxious children (Waters, Pittaway, Mogg, Bradley, \& Pine, 2013). Thus, there is emerging evidence that a positive bias might be protective for child anxiety, although it is noteworthy that attention bias for happy faces has also been linked to externalising problems (Morales, Fu, \& Pérez-Edgar, 2016).

\section{Implications}

Our findings indicate that ABM may not be suitable for young children as attention bias to threat appears to be normative for preschool-aged children. In fact, this might serve as an adaptive function so we should be cautious about trying to change it. Furthermore, for both bias to angry and happy faces, consequences may vary depending on temperament. Given that starting school appears to only affect state anxiety over the initial school transition, it is unlikely that the costs of implementing programmes in community settings to prevent anxiety over this period would be worthwhile. Nevertheless, it remains possible that targeting specific groups of children who are at risk would be valuable, but further work is required to identify which children would benefit and what this intervention should target specifically.

\section{Strengths and limitations}

The study has a number of strengths. Of particular note is the longitudinal design using a normative stressor and the daily reports of anxiety allowing the trajectory of anxiety over the transition to school to be examined. To our knowledge, no previous research has examined whether $\mathrm{BI}$ or attention bias predict state anxiety in response to a stressor in children. Further strengths include the use of an age-appropriate eyetracking task that does not rely on reaction time, as well as the excellent retention rate. Limitations include the selfselecting sample and the reliance on parent-report to assess anxiety. Importantly, however, 
the predictors ( $\mathrm{BI}$, attention bias) did not rely on parent-report, which diminishes concerns about shared method variance. Two further limitations require consideration. First, we evaluated split-half reliability of the eyetracking bias scores and found poor reliability for orientation bias. In light of this, results for orientation bias are not presented and we cannot make conclusions about initial allocation of attention. A number of studies have explicitly examined the reliability of metrics extracted from eyetracking tasks and there is some consensus across studies that orientation bias and other measure of 'early' bias often have very poor reliability (Skinner et al., 2018). In contrast, measures of overall attention or dwell tend to have better reliability, which is consistent with our findings here. The reliability estimate for dwell bias to angry faces was adequate for individual difference research, and consistent with eyetracking reliability in adults (Sears et al., 2019). For dwell bias to happy faces, reliability was weaker and the results that rely on this measure should be considered with this in mind. A final limitation is that our sample was not clinically diagnosed. Longitudinal research aiming to identify opportunities for preventative interventions must begin by assessing putative risk factors before individuals meet criteria for disorder. Ideally, the dependent variable would then be subsequent disorder diagnosis, but for developmental research this requires very large samples and long-term follow-up. An alternative is to have a dependent variable that is a marker of subsequent risk for disorder. For anxiety, both trait anxiety and state anxiety in response to a stressor are informative with regards subsequent risk. The study has therefore been able to evaluate theoretical hypotheses and provide insights into risk mechanisms that are relevant for anxiety disorders using a non-clinical sample.

\section{References}


Abend, R., de Voogd, L., Salemink, E., Wiers, R. W., Pérez-Edgar, K., Fitzgerald, A., White, L.K., Salum, G.A., He, J., Silverman, W.K., Pettit, J.W., Pine, D.S., Bar-Haim, Y. (2018). Association between attention bias to threat and anxiety symptoms in children and adolescents. Depression and Anxiety, 35(3), 229-238.

https://doi.org/10.1002/da.22706

Bar-Haim, Y., Lamy, D., Pergamin, L., Bakermans-Kranenburg, M.J., \& van IJzendoorn, M.H. (2007). Threat-related attentional bias in anxious and nonanxious individuals: a meta-analytic study. Psychological Bulletin, 133(1), 1-24.

https://doi.org/10.1037/0033-2909.133.1.1

Bar-Haim, Y. (2010). Research Review: attention bias modification (ABM): a novel treatment for anxiety disorders. Journal of Child Psychology and Psychiatry, 51(8), 859-870. https://doi.org/10.1111/j.1469-7610.2010.02251.x

Bates, D., Mächler, M., Bolker, B., \& Walker, S. (2014). Fitting linear mixed-effects models using Ime4. arXiv preprint arXiv:1406.5823. https://doi.org/10.18637/jss.v067.i01

Beck, A. T., \& Clark, D. A. (1997). An information processing model of anxiety: Automatic and strategic processes. Behaviour Research and Therapy, 35(1), 49-58. https://doi.org/10.1016/S0005-7967(96)00069-1

Bishop, G., Spence, S. H., \& McDonald, C. (2003). Can parents and teachers provide a reliable and valid report of behavioral inhibition? Child Development, 74(6), 1899-1917. https://doi.org/10.1046/j.1467-8624.2003.00645.x

Britton, J. C., Bar-Haim, Y., Clementi, M. A., Sankin, L. S., Chen, G., Shechner, T., . . P Pine, D. S. (2013). Training-associated changes and stability of attention bias in youth: implications for attention bias modification treatment for pediatric anxiety. Developmental Cognitive Neuroscience, 4, 52-64. https://doi.org/10.1016/j.dcn.2012.11.001

Brown, H. M., Eley, T. C., Broeren, S., MacLeod, C., Rinck, M., Hadwin, J. A., \& Lester, K. J. (2014). Psychometric properties of reaction time based experimental paradigms measuring anxiety-related information-processing biases in children. Journal of Anxiety Disorders, 28(1), 97-107. https://doi.org/10.1016/i.janxdis.2013.11.004

Bucher, A., \& Voss, A. (2019). Judging the mood of the crowd: Attention is focused on happy faces. Emotion, 19(6), 1044-1059. https://doi.org/10.1037/emo0000507

Burris, J. L., Barry-Anwar, R. A., \& Rivera, S. M. (2017). An eye tracking investigation of attentional biases towards affect in young children. Developmental psychology, 53(8), 1418. https://doi.org/10.1037/dev0000345

Chronis-Tuscano, A., Degnan, K. A., Pine, D. S., Perez-Edgar, K., Henderson, H. A., Diaz, Y., . . Fox, N. A. (2009). Stable Early Maternal Report of Behavioral Inhibition Predicts Lifetime Social Anxiety Disorder in Adolescence. Journal of the American Academy of Child \& Adolescent Psychiatry, 48(9), 928-935. https://doi.org/10.1097/CHI.0b013e3181ae09df

Claus, J. A., \& Blackford, J. U. (2012). Behavioural inhibition and risk for developing social anxiety disorder: A meta-analytic study. Journal of the American Academy of Child and Adolescent Psychiatry, 51(10), 1066-1075. https://doi.org/10.1016/j.jaac.2012.08.002

Cole, C. E., Zapp, D. J., Fettig, N. B., \& Pérez-Edgar, K. (2016). Impact of attention biases to threat and effortful control on individual variations in negative affect and social withdrawal in very young children. Journal of Experimental Child Psychology, 141, 210-221. https://doi.org/10.1016/i.jecp.2015.09.012 
Cristea, I. A., Kok, R. N., \& Cuijpers, P. (2015). Efficacy of cognitive bias modification interventions in anxiety and depression: meta-analysis. The British Journal of Psychiatry, 206(1), 7-16. https://doi.org/10.1192/bjp.bp.114.146761

Degnan, K., \& Fox, N. (2007). Behavioral inhibition and anxiety disorders: Multiple levels of a resilience process. Development and Psychopathology, 19(3), 729-746. https://doi.org/10.1017/S0954579407000363

Dink, J., \& Ferguson, B. (2018)._eyetrackingR_. R package version 0.1.8,. $<U R L: h t t p: / / w w w . e y e t r a c k i n g-R . c o m>$

Dodd, H. F., Hudson, J. L., Williams, T., Morris, T., Lazarus, R. S., \& Byrow, Y. (2015). Anxiety and attentional bias in preschool-aged children: An eyetracking study. Journal of abnormal child psychology, 43(6), 1055-1065. https://doi.org/10.1007/s10802-0149962-x

Dudeney, J., Sharpe, L., \& Hunt, C. (2015). Attentional bias towards threatening stimuli in children with anxiety: A meta-analysis. Clinical psychology review, 40, 66-75. https://doi.org/10.1016/i.cpr.2015.05.007

Field, A. P., \& Lester, K. J. (2010). Is there room for 'development' in developmental models of information processing biases to threat in children and adolescents? Clinical Child and Family Psychology Review, 13(4), 315-332. https://doi.org/10.1007/s10567-0100078-8

Fox, N. A., Henderson, H. A., Rubin, K. H., Calkins, S. D., \& Schmidt, L. A. (2001). Continuity and discontinuity ol behavioral inhibition and exuberance: Psychophysiological and behavioral influences across the first four years of life. Child Development, 72(1), 121. https://doi.org/10.1111/1467-8624.00262

Gagne, J. R., Van Hulle, C. A., Aksan, N., Essex, M. J., \& Goldsmith, H. H. (2011). Deriving childhood temperament measures from emotion-eliciting behavioral episodes: Scale construction and initial validation. Psychological Assessment, 23(2), 337. https://doi.org/10.1037/a0021746

Gamble, A. L., \& Rapee, R. M. (2009). The time-course of attentional bias in anxious children and adolescents. Journal of Anxiety Disorders, 23(7), 841-847. https://doi.org/10.1016/j.janxdis.2009.04.001

Goldsmith, H., Reilly, J., Lemery, K., Longley, S., \& Prescott, A. (1999). The laboratory assessment battery: Preschool version (LAB-TAB). Madison: University of Wisconsin.

Grafton, B., MacLeod, C., Rudaizky, D., Holmes, E. A., Salemink, E., Fox, E., \& Notebaert, L. (2017). Confusing procedures with process when appraising the impact of cognitive bias modification on emotional vulnerability. The British Journal of Psychiatry, 211(5), 266-271. https://doi.org/10.1192/bjp.bp.115.176123

Grund, S., Robitzsch, A., \& Luedtke, O. (2019). mitml: Tools for Multiple Imputation in Multilevel Modeling. $R$ package version 0.3-7. https://CRAN.Rproject.org $/$ package $=$ mitml

Henderson, H. A., Pine, D. S., \& Fox, N. A. (2015). Behavioral inhibition and developmental risk: a dual-processing perspective. Neuropsychopharmacology, 40(1), 207. https://doi.org/10.1038/npp.2014.189

Hudson, J. L., \& Dodd, H. F. (2012). Informing early intervention: Preschool predictors of anxiety disorders in middle childhood. PLOS ONE, 7(8), e42359. https://doi.org/10.1371/journal.pone.0042359 
Hudson, J. L., Murayama, K., Meteyard, L., Morris, T., \& Dodd, H. F. (2018). Early childhood predictors of anxiety in early adolescence. Journal of Abnormal Child Psychology, 47(7), 1-13. https://doi.org/10.1007/s10802-018-0495-6

Kagan, J., Reznick, J. S., Clarke, C., Snidman, N., \& Garciacoll, C. (1984). Behavioral Inhibition to the unfamiliar. Child Development, 55(6), 2212-2225. https://doi.org/10.2307/1129793

Kim, J., Klein, D. N., Olino, T. M., Dyson, M. W., Dougherty, L. R., \& Durbin, C. E. (2011). Psychometric properties of the Behavioral Inhibition Questionnaire in preschool children. Journal of Personality Assessment, 93(6), 545-555. https://doi.org/10.1080/00223891.2011.608756

Kruijt, A., Parsons, S., Fox, E. (2018). A meta-analysis of bias at baseline in RCTs of attention bias modification: No evidence for dot-probe bias towards threat in clinical anxiety and PTSD. Journal of Abnormal Psychology, 128(6), 563-573. https://doi.org/10.1037/abn0000406

Kuznetsova, A., Brockhoff, P. B., \& Christensen, R. H. B. (2017). ImerTest package: tests in linear mixed effects models. Journal of Statistical Software, 82(13). https://doi.org/10.18637/jss.v082.i13

Lisk, S., Vaswani, A., Linetzky, M., Bar-Haim, Y., \& Lau, J. Y. F. (2019). Systematic Review and Meta-Analysis: Eye-Tracking of Attention to Threat in Child and Adolescent Anxiety. Journal of the American Academy of Child and Adolescent Psychiatry, 59(1), 88-91. https://doi.org/10.1016/j.jaac.2019.06.006

Liu, P., \& Pérez-Edgar, K. E. (2019). Developmental Pathways from Early Behavioral Inhibition to Later Anxiety: An Integrative Review of Developmental Psychopathology Research and Translational Implications. Adolescent Research Review, 4(1), 45-58.

https://doi.org/10.1007/s40894-018-0092-5

LoBue, V. (2009). More than just another face in the crowd: Superior detection of threatening facial expressions in children and adults. Developmental Science, 12(2), 305-313. https://doi.org/10.1111/i.1467-7687.2008.00767.x

LoBue, V., \& DeLoache, J. S. (2010). Superior detection of threat-relevant stimuli in infancy. Developmental Science, 13(1), 221-228. https://doi.org/10.1111/j.14677687.2009.00872.x

LoBue, V., \& Thrasher, C. (2015). The Child Affective Facial Expression (CAFE) set: Validity and reliability from untrained adults. Frontiers in Psychology, 5, 1532. https://doi.org/10.3389/fpsyg.2014.01532

MacLeod, C., \& Clarke, P. J. (2015). The attentional bias modification approach to anxiety intervention. Clinical Psychological Science, 3(1), 58-78. https://doi.org/10.1177/2167702614560749

MacLeod, C., \& Hagan, R. (1992). Individual differences in the selective processing of threatening information, and emotional responses to a stressful life event. Behaviour Research and Therapy, 30(2), 151-161. https://doi.org/10.1016/00057967(92)90138-7

Mathews, A., \& Mackintosh, B. (1998). A cognitive model of selective processing in anxiety. Cognitive Therapy and Research, 22(6), 539-560. https://doi.org/10.1023/A:1018738019346

Mathews, A., \& MacLeod, C. (2002). Induced processing biases have causal effects on anxiety. Cognition \& Emotion, 16(3), 331-354. https://doi.org/10.1080/02699930143000518 
Mogg, K., \& Bradley, B. P. (1998). A cognitive-motivational analysis of anxiety. Behaviour Research and Therapy, 36(9), 809-848. https://doi.org/10.1016/S00057967(98)00063-1

Mogg, K., \& Bradley, B. P. (2016). Anxiety and attention to threat: Cognitive mechanisms and treatment with attention bias modification. Behaviour Research and Therapy, 87, 76108. https://doi.org/10.1016/j.brat.2016.08.001

Mogg, K., \& Bradley, B. P. (2018). Anxiety and threat-related attention: Cognitivemotivational framework and treatment. Trends in Cognitive Sciences, 22(3), 225-240. https://doi.org/10.1016/j.tics.2018.01.001

Mogg, K., Waters, A. M., \& Bradley, B. P. (2017). Attention bias modification (ABM): Review of effects of multisession $A B M$ training on anxiety and threat-related attention in high-anxious individuals. Clinical Psychological Science, 5(4), 698-717. https://doi.org/10.1177/2167702617696359

Morales, S., Fu, X., \& Pérez-Edgar, K. E. (2016). A developmental neuroscience perspective on affect-biased attention. Developmental Cognitive Neuroscience, 21, 26-41. https://doi.org/10.1016/j.den.2016.08.001

Morales, S., Pérez-Edgar, K. E., \& Buss, K. A. (2015). Attention biases towards and away from threat mark the relation between early dysregulated fear and the later emergence of social withdrawal. Journal of Abnormal Child Psychology, 43(6), 1067-1078. https://doi.org/10.1007/s10802-014-9963-9

National Institute of Mental Health. Development and Definitions of the RDoc Domains and Constructs (2011). Available from: https://www.nimh.nih.gov/research/researchfunded-by-nimh/rdoc/definitions-of-the-rdoc-domains-and-constructs.shtml

Nozadi, S. S., Troller-Renfree, S., White, L. K., Frenkel, T., Degnan, K. A., Bar-Haim, Y., . . Fox, N. A. (2016). The moderating role of attention biases in understanding the link between behavioral inhibition and anxiety. Journal of Experimental Psychopathology, 7(3), 451-465. https://doi.org/10.5127/jep.052515

Osinsky, R., Losch, A., Hennig, J., Alexander, N., \& Macleod, C. (2012). Attentional bias to negative information and 5-HTTLPR genotype interactively predict students' emotional reactivity to first university semester. Emotion, 12(3), 460-469. doi:10.1037/a0026674 https://doi.org/10.1037/a0026674

Parsons, S. (2019). splithalf: Robust estimates of split half reliability (R package Version 5) [Computer software]. doi:10.6084/m9.figshare.5559175.v5

Parsons, S., Kruijt, A.-W., \& Fox, E. (2019). Psychological Science Needs a Standard Practice of Reporting the Reliability of Cognitive-Behavioral Measurements. Advances in Methods and Practices in Psychological Science, 2(4), 378-395.

https://doi.org/10.1177/2515245919879695

Perez-Edgar, K., McDermott, J. N., Korelitz, K., Degnan, K. A., Curby, T. W., Pine, D. S., \& Fox, N. A. (2010). Patterns of sustained attention in infancy shape the developmental trajectory of social behavior from toddlerhood through adolescence. Developmental Psychology, 46(6), 1723-1730. https://doi.org/10.1037/a0021064

Perez-Edgar, K., Reeb-Sutherland, B. C., McDermott, J. M., White, L. K., Henderson, H. A., Degnan, K. A., . . Fox, N. A. (2011). Attention biases to threat link behavioral inhibition to social withdrawal over time in very young children. Journal of Abnormal Child Psychology, 39(6), 885-895. https://doi.org/10.1007/s10802-011-9495-5

Pettit, J. W., Bechor, M., Rey, Y., Vasey, M. W., Abend, R., Pine, D. S., . . Silverman, W. K. (2019). A Randomized Controlled Trial of Attention Bias Modification Treatment in 
Youth With Treatment-Resistant Anxiety Disorders. Journal of the American Academy of Child and Adolescent Psychiatry 59(1), 157-165.

https://doi.org/10.1016/i.jaac.2019.02.018

Price, R. B., Kuckertz, J. M., Siegle, G. J., Ladouceur, C. D., Silk, J. S., Ryan, N. D., . . Amir, N. (2015). Empirical recommendations for improving the stability of the dot-probe task in clinical research. Psychological Assessment, 27(2), 365.

https://doi.org/10.1037/pas0000036

Raymond, J.G., Steele, J.D., Series, P. (2017). Modeling Trait Anxiety: From Computational Processes to Personality. Frontiers in Psychiatry, 8, 1-19.

https://doi.org/10.3389/fpsyt.2017.00001

Rodebaugh, T.L., Scullin, R.B., Langer, J.K., Dixon, D.J., Huppert, J.D., Bernstein, A., Zvielli, A., \& Lenze, E.J. (2016). Unreliability as a threat to understanding psychopathology: The cautionary tale of attentional bias. Journal of Abnormal Psychology, 125(6), 840-851. https://doi/org/10.1037/abn0000184

Salum, G. A., Mogg, K., Bradley, B. P., Gadelha, A., Pan, P., Tamanaha, A. C., . . Pine, D. S. (2013). Threat bias in attention orienting: evidence of specificity in a large community-based study. Psychological Medicine, 43(4), 733-745. https://doi.org/10.1017/S0033291712001651

Sears, C., Quigley, L., Fernandez, A., Newman, K., \& Dobson, K. (2019). The reliability of attentional biases for emotional images measured using a free-viewing eye-tracking paradigm. Behavioural Research Methods, 51(6), 2748-2760.

https://doi.org/10.3758/s13428-018-1147-z

Schmidtendorf, S., Wiedau, S., Asbrand, J., Tuschen-Caffier, B., \& Heinrichs, N. (2018). Attentional Bias in Children with Social Anxiety Disorder. Cognitive Therapy and Research, 42(3), 273-288. https://doi.org/10.1007/s10608-017-9880-7

Shechner, T., Britton, J. C., Pérez-Edgar, K., Bar-Haim, Y., Ernst, M., Fox, N. A., . . Pine, S. D. (2012). Attention biases, anxiety, and development: Toward or away from threats or rewards? Depression and Anxiety, 29(4), 282-284. https://doi.org/10.1002/da.20914

Shechner, T., Jarcho, J. M., Britton, J. C., Leibenluft, E., Pine, D. S., \& Nelson, E. E. (2013). Attention bias of anxious youth during extended exposure of emotional face pairs: An eye-tracking study. Depression and Anxiety, 30(1), 14-21. https://doi.org/10.1002/da.21986

Skinner, I.W., Hübscher, M., Moseley, G.L., Lee, H., Wand, B.M., Traeger, A.C., Gustin, S.M., \& McAuley, J.H. (2018). The reliability of eyetracking to assess attentional bias to threatening words in healthy individuals. Behavior Research, 50(5), 1778-1792. https://doi.org/10.3758/s13428-017-0946-y

Spence, S. H., Rapee, R., McDonald, C., \& Ingram, M. (2001). The structure of anxiety symptoms among preschoolers. Behaviour Research and Therapy, 39(11), 1293-1316. https://doi.org/10.1016/S0005-7967(00)00098-X

Van Bockstaele, B., Verschuere, B., Tibboel, H., De Houwer, J., Crombez, G., \& Koster, E. H. (2014). A review of current evidence for the causal impact of attentional bias on fear and anxiety. Psychological Bulletin, 140(3), 682. https://doi.org/10.1037/a0034834

van Buuren, S., \& Groothuis-Oudshoorn, K. (2011). mice: Multivariate Imputation by Chained Equations in R. 2011, 45(3), 67. https://doi.org/10.18637/jss.v045.i03

Van Damme, S., \& Crombez, G. (2009). Measuring attentional bias to threat in children and adolescents: A matter of speed? Journal of Behaviour Therapy and Experimental Psychiatry, 40(2), 344-351. https://doi.org/10.1016/i.jbtep.2009.01.004 
Van Den Hout, M., Tenney, N., Huygens, K., Merckelbach, H., \& Kindt, M. (1995). Responding to subliminal threat cues is related to trait anxiety and emotional vulnerability: A successful replication of MacLeod and Hagan (1992). Behaviour Research and Therapy, 33(4), 451-454. https://doi.org/10.1016/0005-7967(94)00062-O

Waechter, S., Nelson, A. L., Wright, C., Hyatt, A., \& Oakman, J. (2014). Measuring attentional bias to threat: Reliability of dot probe and eye movement indices. Cognitive Therapy and Research, 38(3), 313-333. https://doi.org/10.1007/s10608-013-9588-2

Waters, A., Bradley, B., \& Mogg, K. (2014). Biased attention to threat in paediatric anxiety disorders (generalized anxiety disorder, social phobia, specific phobia, separation anxiety disorder) as a function of 'distress' versus 'fear'diagnostic categorization. Psychological Medicine, 44(3), 607-616. https://doi.org/10.1017/S0033291713000779

Waters, A., Pittaway, M., Mogg, K., Bradley, B., \& Pine, D. (2013). Attention training towards positive stimuli in clinically anxious children. Developmental Cognitive Neuroscience, 4, 77-84. https://doi.org/10.1016/i.dcn.2012.09.004

White, L. K., Degnan, K. A., Henderson, H. A., Pérez-Edgar, K., Walker, O. L., Shechner, T., . . . Fox, N. A. (2017). Developmental relations among behavioral inhibition, anxiety, and attention biases to threat and positive information. Child Development, 88(1), 141155. https://doi.org/10.1111/cdev.12696

Wickham, H. (2017). The tidyverse. R package ver. 1.2.1, https://CRAN.Rproject.org/package=tidyverse.

Williams, J. M. G., Watts, F. N., MacLeod, C., \& Mathews, A. (1988). Cognitive psychology and emotional disorders: John Wiley \& Sons. 
Table 1

Means, standard deviations, and correlations with confidence intervals

\begin{tabular}{|c|c|c|c|c|c|c|c|}
\hline Variable & $M$ & $S D$ & 1 & 2 & 3 & 4 & 5 \\
\hline $\begin{array}{l}\text { 1. Dwell Bias } \\
\text { Angry Faces }\end{array}$ & 0.14 & 0.08 & - & & & & \\
\hline $\begin{array}{l}\text { 2. Dwell Bias } \\
\text { Happy Faces }\end{array}$ & 0.03 & 0.06 & $\begin{array}{c}.29 * * \\
{[.14, .42]}\end{array}$ & & & & \\
\hline 3. Baseline PAS & 23.79 & 14.47 & $\begin{array}{c}-.12 \\
{[-.26, .04]}\end{array}$ & $\begin{array}{l}-.07 \\
{[-.22, .08]}\end{array}$ & & & \\
\hline 4. Follow-up PAS & 22.43 & 13.65 & $\begin{array}{l}-.06 \\
{[-.22, .10]}\end{array}$ & $\begin{array}{l}-.09 \\
{[-.25, .07]}\end{array}$ & $\begin{array}{c}.76^{* *} \\
{[.69, .82]}\end{array}$ & & \\
\hline 5. $\mathrm{BI}$ & -0.01 & 0.81 & $\begin{array}{c}.01 \\
{[-.15, .16]}\end{array}$ & $\begin{array}{l}-.02 \\
{[-.17, .14]}\end{array}$ & $\begin{array}{c}.54^{* *} \\
{[.42, .64]}\end{array}$ & $\begin{array}{c}.46^{* *} \\
{[.32, .58]}\end{array}$ & \\
\hline $\begin{array}{l}\text { 6. Average Daily } \\
\text { Anxiety Rating }\end{array}$ & 1.64 & 1.39 & $\begin{array}{c}-.06 \\
{[-.21, .09]}\end{array}$ & $\begin{array}{c}-.11 \\
{[-.26, .04]}\end{array}$ & $\begin{array}{c}.45^{* *} \\
{[.32, .56]}\end{array}$ & $\begin{array}{c}.54 * * \\
{[.42, .64]}\end{array}$ & $\begin{array}{r}.35^{* *} \\
{[.21, .48]}\end{array}$ \\
\hline
\end{tabular}

Note. PAS = Preschool Anxiety Scale; $\mathrm{BI}=$ Behavioural Inhibition.

Values in square brackets indicate the $95 \% \mathrm{Cls}$. 
$*$ indicates $p<.05 . * *$ indicates $p<.01$. 
Table 2

Regression results for cross-sectional analyses predicting anxiety scores on the Preschool Anxiety Scale (PAS).



Note. $\mathrm{BI}=$ Behavioural Inhibition. A.Bias $=$ Dwell bias for angry faces. H.Bias = Dwell bias for

Happy faces.

$*$ indicates $p<.05 . * *$ indicates $p<.01$. 
Table 3

Regression results for longitudinal analyses predicting anxiety scores on the Preschool Anxiety Scale (PAS) at follow-up.

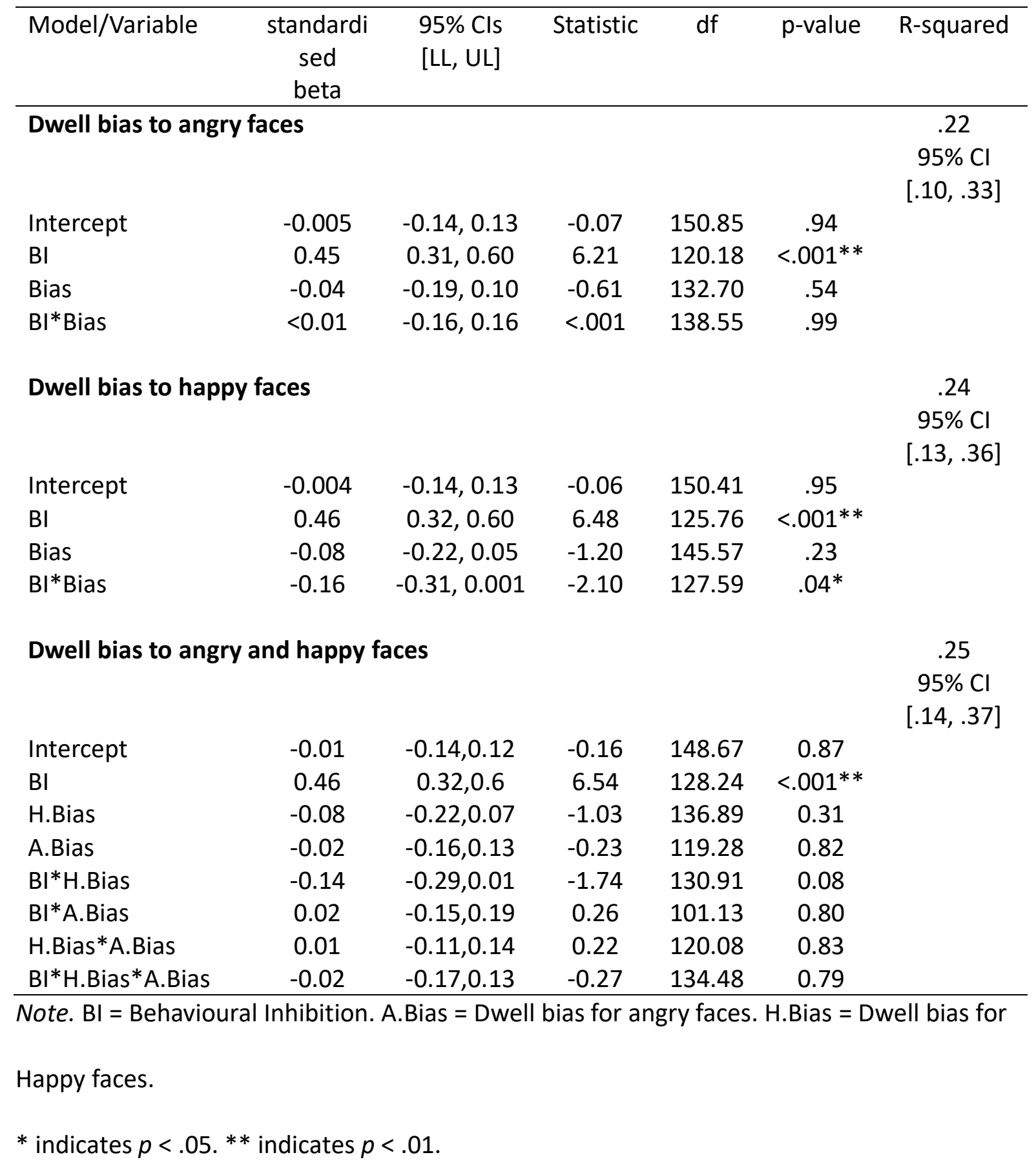


Table 4

Coefficients of Final Model for Dwell Bias for Angry Faces

\begin{tabular}{|c|c|c|c|c|}
\hline Term & Estimate & $p$ & 95\% C.I.S & $\begin{array}{c}\text { Random } \\
\text { Effect } \\
\text { Variance }\end{array}$ \\
\hline (Intercept) & 1.64 & $<.001 * *$ & {$[1.44,1.83]$} & 1.57 \\
\hline $\mathrm{BI}$ & 0.47 & $<.001 * *$ & {$[0.27,0.67]$} & - \\
\hline Bias & -0.09 & 0.37 & {$[-0.30,0.11]$} & - \\
\hline Time & -0.44 & $<.001 * *$ & {$[-0.50,-0.38]$} & - \\
\hline Time $^{2}$ & $<0.01$ & 0.997 & {$[-0.06,0.06]$} & - \\
\hline BI*Bias & -0.01 & 0.97 & {$[-0.26,0.25]$} & - \\
\hline BI*Time & -0.02 & 0.61 & {$[-0.08,0.05]$} & - \\
\hline BI*Time ${ }^{2}$ & -0.05 & 0.12 & {$[-0.12,0.03]$} & - \\
\hline Bias*Time & 0.02 & 0.96 & {$[-0.07,0.07]$} & - \\
\hline Bias*Time $^{2}$ & 0.02 & 0.55 & {$[-0.05,0.08]$} & - \\
\hline BI*Bias*Time & -0.05 & 0.25 & {$[-0.12,0.03]$} & - \\
\hline BI*Bias*Time ${ }^{2}$ & 0.12 & $0.005^{* *}$ & {$[0.04,0.20]$} & - \\
\hline Residual Variance & . & . & . & 2.29 \\
\hline
\end{tabular}

Note. $\mathrm{BI}=$ Behavioural Inhibition.

$*$ indicates $p<.05 . * *$ indicates $p<.01$.

Table 5

Coefficients of Final Model for Dwell Bias for Happy Faces

Random

Effect

\begin{tabular}{lcccc} 
Term & Estimate & $p$ & $95 \%$ C.I.s & Variance \\
\hline (Intercept) & 1.64 & $<.001^{* *}$ & {$[1.44,1.83]$} & 1.53 \\
BI & 0.47 & $<.001^{* *}$ & {$[0.27,0.67]$} & - \\
Bias & -0.13 & 0.20 & {$[-0.33,0.07]$} & - \\
Time & -0.44 & $<.001^{* *}$ & {$[-0.50,-0.38]$} & - \\
BI*Bias & -0.20 & 0.07 & {$[-0.42,0.01]$} & - \\
BI*Time & -0.02 & 0.53 & {$[-0.09,0.05]$} & - \\
Bias*Time & 0.04 & 0.30 & {$[-0.03,0.10]$} & - \\
BI*Bias*Time & 0.11 & $.002^{* *}$ & {$[0.04,0.18]$} & - \\
Residual Variance &. &. &. & 2.30 \\
\hline
\end{tabular}


Note. $\mathrm{BI}=$ Behavioural Inhibition .

$*$ indicates $p<.05 . * *$ indicates $p<.01$.

Table 6

Coefficients of Model for Dwell Bias with Angry and Happy Faces

\begin{tabular}{|c|c|c|c|c|}
\hline Term & Estimate & $p$ & 95\% C.I.s & $\begin{array}{c}\text { Random } \\
\text { Effect } \\
\text { Variance }\end{array}$ \\
\hline (Intercept) & 1.63 & $<.001 * *$ & {$[1.42,1.82]$} & 1.56 \\
\hline $\mathrm{BI}$ & 0.47 & $<.001 * *$ & {$[0.26,0.66]$} & - \\
\hline Angry Bias & -0.04 & .69 & {$[-0.25,0.17]$} & - \\
\hline Happy Bias & -0.11 & .31 & {$[-0.32,0.1]$} & \\
\hline Time & -0.44 & $<.001 * *$ & {$[-0.5,-0.37]$} & - \\
\hline Time $^{2}$ & 0.01 & .82 & {$[-0.05,0.07]$} & \\
\hline BI*Angry Bias & 0.01 & .95 & {$[-0.24,0.26]$} & - \\
\hline BI*Happy Bias & -0.20 & .09 & {$[-0.42,0.03]$} & \\
\hline Angry Bias*Happy Bias & 0.08 & .39 & {$[-0.1,0.27]$} & \\
\hline Bl*Time & -0.02 & .55 & {$[-0.08,0.04]$} & - \\
\hline BI*Time ${ }^{2}$ & -0.05 & .13 & {$[-0.11,0.01]$} & \\
\hline Angry Bias*Time & -0.02 & .57 & {$[-0.09,0.05]$} & - \\
\hline Angry Bias*Time ${ }^{2}$ & 0.01 & .82 & {$[-0.06,0.07]$} & \\
\hline Happy Bias*Time & 0.05 & .17 & {$[-0.02,0.12]$} & \\
\hline Happy Bias*Time ${ }^{2}$ & 0.06 & .10 & {$[-0.01,0.13]$} & \\
\hline BI*Angry Bias*Happy Bias & -0.10 & .42 & {$[-0.33,0.13]$} & \\
\hline BI*Angry Bias*Time & -0.06 & .15 & {$[-0.13,0.02]$} & - \\
\hline BI*Angry Bias*Time ${ }^{2}$ & 0.11 & $<.001 * *$ & {$[0.03,0.19]$} & \\
\hline BI*Happy Bias*Time & 0.12 & $<.001 * *$ & {$[0.04,0.19]$} & \\
\hline BI*Happy Bias*Time ${ }^{2}$ & 0.03 & .50 & {$[-0.05,0.1]$} & \\
\hline Angry Bias*Happy Bias*Time & 0.01 & .74 & {$[-0.05,0.07]$} & \\
\hline Angry Bias*Happy Bias*Time ${ }^{2}$ & -0.01 & .78 & {$[-0.06,0.05]$} & \\
\hline BI*Angry Bias*Happy Bias*Time & -0.03 & .51 & {$[-0.1,0.05]$} & \\
\hline BI*Angry Bias*Happy Bias*Time ${ }^{2}$ & -0.05 & .23 & {$[-0.12,0.02]$} & \\
\hline Residual Variance & . & . & . & 2.28 \\
\hline
\end{tabular}

Note: This model includes quadratic and linear time to align with the highest order model when angry bias and happy bias were examined alone. 
$\mathrm{BI}=$ Behavioural Inhibition.

$*$ indicates $p<.05 . * *$ indicates $p<.01$. 